\title{
Corticolimbic Mechanisms of Behavioral Inhibition under Threat of Punishment
}

\author{
미eroen P.H. Verharen, ${ }^{1,2}$ Mauri W. van den Heuvel, ${ }^{1}$ Mieneke Luijendijk, ${ }^{1}$ 느ouk J.M.J. Vanderschuren, ${ }^{2 *}$ \\ and $D_{\text {Roger A.H. Adan }}^{1,3 *}$ \\ ${ }^{1}$ Brain Center Rudolf Magnus, Department of Translational Neuroscience, University Medical Center Utrecht, 3584CG Utrecht, The Netherlands, \\ ${ }^{2}$ Department of Animals in Science and Society, Division of Behavioural Neuroscience, Faculty of Veterinary Medicine, Utrecht University, 3584 CM, \\ Utrecht, The Netherlands, and ${ }^{3}$ Institute of Physiology and Neuroscience, Sahlgrenska Academy at the University of Gothenburg, 413-90, Gothenburg, \\ Sweden
}

Being able to limit the pursuit of reward to prevent negative consequences is an important expression of behavioral inhibition. Everyday examples of an inability to exert such control over behavior are the overconsumption of food and drugs of abuse, which are important factors in the development of obesity and addiction, respectively. Here, we use a behavioral task that assesses the ability of male rats to exert behavioral restraint at the mere sight of palatable food during the presentation of an audiovisual threat cue to investigate the corticolimbic underpinnings of behavioral inhibition. We demonstrate a prominent role for the medial prefrontal cortex in the exertion of control over behavior under threat of punishment. Moreover, task engagement relies on function of the ventral striatum, whereas the basolateral amygdala mediates processing of the threat cue. Together, these data show that inhibition of reward pursuit requires the coordinated action of a network of corticolimbic structures.

Key words: corticolimbic; inhibition; punishment; reward

\section{Significance Statement}

There is a need for translational models that allow to dissect mechanisms underlying the processes involved in controlling behavior. In this study, we present a novel behavioral task that assesses the ability of rats to exert behavioral restraint over the consumption of a visually present sucrose pellet during the presentation of an audiovisual threat cue. This task requires relatively little behavioral training and it discerns distinct behavioral impairments, including a failure to retrieve stimulus value, a reduced task engagement, and compromised inhibition of behavior. Using pharmacological inactivations of different regions of the corticolimbic system of the rat, we demonstrate dissociable roles for the prefrontal cortex, amygdala, and striatum in inhibition of reward pursuit under threat of punishment.

\section{Introduction}

In a world where food is abundantly available, it can be hard to resist the temptation to eat highly palatable, yet unhealthy foods, while being aware of the negative health consequences this may have. As such, a healthy lifestyle requires one to control the urge

\footnotetext{
Received Nov. 6, 2018; revised Dec. 21, 2018; accepted Jan. 28, 2019.

Author contributions: J.P.H.V., L.J.M.J.V., and R.A.H.A. designed research; J.P.H.V., M.W.v.d.H., and M.L. performed research; J.P.H.V. and M.W.v.d.H. analyzed data; J.P.H.V. wrote the first draft of the paper; J.P.H.V., L.J.M.J.V., and R.A.H.A. edited the paper.

This work was supported by the European Union Seventh Framework Programme under Grant 607310 (Nudge-IT) and the Netherlands Organization for Scientific Research (NWO) under project 912.14 .093 (Shining light on loss of control).

The authors declare no competing financial interests.

*L.J.M.J.V. and R.A.H.A. contributed equally to this work.

Correspondence should be addressed to Roger A. H. Adan at r.a.h.adan@umcutrecht.nl.

https://doi.org/10.1523/JNEUROSCI.2814-18.2019

Copyright $\odot 2019$ the authors
}

to eat tasty foods. This can be especially challenging during dieting, when the body is in a negative energy state, and food cues are more salient than usual (van der Plasse et al., 2015). Accordingly, reduced behavioral inhibition has shown to be an important factor in the development and maintenance of overweight in children (Nederkoorn et al., 2006) and adults (Nederkoorn et al., 2010).

In addition to its role in eating and dieting, deficiencies in inhibitory control have been implicated in a wide variety of maladaptive behaviors, ranging from failures in everyday life, like an inability to attain goals, to mental disorders, like substance addiction, attention-deficit/hyperactivity disorder (ADHD), and obsessive-compulsive disorder (Winstanley et al., 2006; Bari and Robbins, 2013; Dalley and Robbins, 2017). Behavioral inhibition is generally assumed to be a multifaceted phenomenon, whereby a distinction can be made between control over actions and control over choices and decisions (Pattij and Vanderschuren, 2008; 
Dalley and Robbins, 2017). These processes have been widely studied using laboratory tasks of impulsivity, which have tremendously progressed our understanding of the neural circuits involved in behavioral control (Pattij and Vanderschuren, 2008; Dalley et al., 2011; Dalley and Robbins, 2017). Indeed, the distinction between choices, decision, and actions is theoretically and mechanistically useful, but many everyday cases in which control over behavior is compromised reflect a combination of these types of impulsivity. For example, an inability to resist a tasty dessert during dieting can sometimes be initiated by a thoughtless walk to the fridge, but during consumption, many decision moments take place in which one can reflect on his or her behavior and consider the consequences of continued eating in the short- and long-term.

In an attempt to capture behavioral inhibition in an ecologically valid fashion, we have developed a behavioral task in rats that measures the ability of the animals to inhibit the urge to consume a highly palatable food reward when a stimulus is presented that signals that sugar retrieval will be punished with a mild electric foot shock. Such a threat puts the animals in a conflict situation, in which a natural approach response to food competes with the natural avoidance response to danger. As such, our task assesses inhibitory control over the innate desire to obtain a readily available reward.

Adaptive inhibition of behavior is thought to rely on functional activity in a network of regions including the prefrontal cortex (PFC), ventral striatum, and amygdala, which has been implicated in the processing of emotionally relevant cues, the selection of appropriate behavioral strategies and the transmission of such strategies into goal-directed behavior (Cardinal et al., 2002; Dalley et al., 2011; Floresco, 2015; Janak and Tye, 2015). Therefore, we tested how pharmacological inactivation of these brain structures altered behavior in this task. We hypothesized that inactivation of these structures would lead to marked, but behaviorally dissociable impairments in task performance.

\section{Materials and Methods}

Animals. A total of 121 male Long-Evans rats (Rj:Orl, Janvier Labs), weighing 250-300 g at the start of the experiment, were used for this study. Animals were kept on a $12 \mathrm{~h}$ reversed day/night cycle (lights off at 8:00 A.M.). Animals were socially housed before surgery, but singly after surgery to prevent damage to the head implant. Experimental procedures were approved by the Animal Ethics Committee of Utrecht University and the Dutch Central Animal Testing Committee and they were conducted in agreement with Dutch (Wet op de Dierproeven, 2014) and European legislation (2010/63/EU).

Surgeries. For placement of the guide cannulae, animals were anesthetized with an intramuscular injection of a mixture of $0.315 \mathrm{mg} / \mathrm{kg}$ fentanyl and $10 \mathrm{mg} / \mathrm{kg}$ fluanisone (Hypnorm, Janssen Pharmaceutica), and placed in a stereotaxic apparatus (David Kopf Instruments). An incision was made along the midline of the skull, and two small craniotomies were made bilaterally above the brain region-of-interest. The following coordinates were used for placement of the guide cannulae (in $\mathrm{mm}$ ): prelimbic cortex: $\mathrm{AP}+3.2, \mathrm{ML} \pm 0.6, \mathrm{DV}-2.6$ from skull; infralimbic cortex: $\mathrm{AP}+3.2, \mathrm{ML} \pm 0.6, \mathrm{DV}-4.3$ from skull; medial orbitofrontal cortex: AP $+4.4, \mathrm{ML} \pm 0.6, \mathrm{DV}-3.8$ from skull; anterior cingulate cortex: $\mathrm{AP}+2.0$, $\mathrm{ML} \pm 0.6, \mathrm{DV}-2.2$ from skull; lateral orbitofrontal cortex: $\mathrm{AP}+3.6, \mathrm{ML}$ $\pm 2.6, \mathrm{DV}-3.7$ from skull under a $5^{\circ}$ angle; basolateral amygdala: AP $-3.0, \mathrm{ML} \pm 5.0, \mathrm{DV}-7.5$ from skull; ventral striatum (core): $\mathrm{AP}+1.2$, $\mathrm{ML} \pm 2.1, \mathrm{DV}-6.3$ from skull under a $5^{\circ}$ angle; ventral striatum (shell): $\mathrm{AP}+1.2, \mathrm{ML} \pm 2.7, \mathrm{DV}-7.0$ from skull under a $10^{\circ}$ angle; dorsomedial striatum: $\mathrm{AP}+1.2, \mathrm{ML} \pm 2.3, \mathrm{DV}-4.1$ from skull under a $5^{\circ}$ angle; dorsolateral striatum: $\mathrm{AP}+1.2, \mathrm{ML} \pm 3.4, \mathrm{DV}-4.1$ from skull; olfactory cortex: $\mathrm{AP}+3.6, \mathrm{ML} \pm 2.2, \mathrm{DV}-4.4$ from skull.
For the brain regions of the medial PFC (prelimbic, infralimbic, medial orbitofrontal, and anterior cingulate cortices), $23 \mathrm{G}$ bilateral guide cannulae were used that had a double protrusion, spaced $1.2 \mathrm{~mm}$ apart (Plastics One). For the other regions (lateral orbitofrontal cortex, basolateral amygdala, olfactory cortex, striatum), two $23 \mathrm{G}$ single-guide cannulae (Plastics One) were placed bilaterally.

Guide cannulae were lowered to the desired coordinates, secured with screws, dental glue (C\&B Metabond, Parkell) and dental cement, and the skin around the cemented cap was sutured. Dummy cannulae were placed inside the guide cannulae. Postsurgery, the animals were injected with $5 \mathrm{mg} / \mathrm{kg}$ carprofen for pain relief $(1 \times / \mathrm{d}$, for $3 \mathrm{~d}$, s.c. $)$ and saline for rehydration (10 $\mathrm{ml}$ once, s.c.), and they were allowed to recover for $7 \mathrm{~d}$ before behavioral training continued.

Experimental procedures. Behavioral testing took place during the dark phase of the reversed $12 \mathrm{~h}$ day/night cycle. The task was conducted in operant conditioning chambers $(31 \times 24 \times 21 \mathrm{~cm}$; MedPC, Med Associates), placed in sound-attenuating cubicles. The chamber contained a shock grid floor, a $28 \mathrm{~V} / 100 \mathrm{~mA}$ houselight, and in the right wall a food port with infrared movement detection, two $28 \mathrm{~V} / 100 \mathrm{~mA}$ cue lights (flanking the food port), and a tone generator $(4500 \mathrm{~Hz})$. A pellet dispenser delivered $45 \mathrm{mg}$ sucrose pellets into the food port (SP; 5TUL; TestDiet). Operant chambers were controlled by MedPC software vIV (Med Associates). Animals were kept on food restriction during the training phase $(\sim 4 \mathrm{~g}$ chow per $100 \mathrm{~g}$ body weight) and always had $\mathrm{ad}$ libitum access to water in their home cage. After successful training, animals received ad libitum chow. However, before behavioral testing, animals were food restricted for $\sim 3 \mathrm{~h}$.

Task. A session consisted of 60 trials of $40 \mathrm{~s}$ each. At the start of every trial, one sucrose pellet was delivered into the food port, regardless of trial type (Fig. 1a). The trials were pseudorandomly distributed so that 30 trials were assigned as "no-stimulus trials", and the remaining 30 trials were assigned as "stimulus trials". This order of trials was the same for all the animals, so that a larger cohort of animals could be tested simultaneously in the same room, without leakage of stimulus sound between the boxes. The house light was illuminated for the entire length of the session.

All trials started with the delivery of a sucrose pellet into the food port. During no-stimulus trials, the animals were allowed to enter the food port (i.e., consume the pellet) directly, which was detected by disruption of the infrared photobeam in the port. During stimulus trials, pellet delivery coincided with the onset of a continuous tone and cue light stimulus, which lasted for $12 \mathrm{~s}$, functioning as a threat signal to the animal. That is, the tone and light cue indicated that the animals had to wait with food port entry (and pellet consumption) until stimulus termination. If the animal managed to wait for $12 \mathrm{~s}$, it could freely enter the food port and consume the sugar without scheduled consequences; this was called a "success" trial. Food port entry during the stimulus, however, terminated the stimulus and delivered a $0.3 \mathrm{~s}$ foot shock to the animal; this was termed a "shock" trial. The intensity of this foot shock was determined during the training phase for each animal individually, but it was kept constant for each animal during the experiment (median foot shock intensity $0.50 \mathrm{~mA}$; Fig. $1 a$ ).

During the task, MedPC software recorded, for each trial, the type of trial (stimulus or no-stimulus), the response of the animal (pellet retrieved or not, and for stimulus trials if the trial was punished or not), the timestamp of the pellet drop, and the timestamp of the response of the animal. Because latencies of pellet retrieval were usually not normally distributed within a session, the median latency for each trial type per session, per animal was used in the analysis. For the latency of pellet retrieval in success trials, we subtracted the $12 \mathrm{~s}$ waiting period from the latency, thus showing the latency of pellet retrieval from stimulus offset, rather than from pellet delivery.

When the animal did not enter the food port (and consume the pellet) during a trial (i.e., within $40 \mathrm{~s}$ ), it was counted as an omission, and this prevented further pellet delivery (and hence pellet accumulation in the food port) until the next food port entry. To control for these omissions, we computed a shock index, which is the number of shock trials as a fraction of the number of shock + success trials. In other words, this index is a measure for the amount of stimulus trials during which the animal entered the food port during stimulus presentation, corrected for 
a Task setup
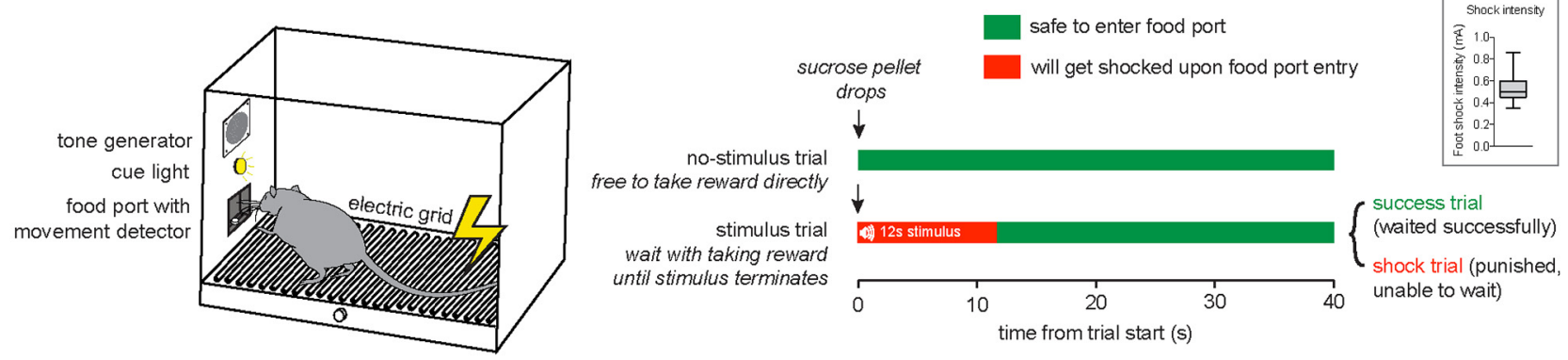

b

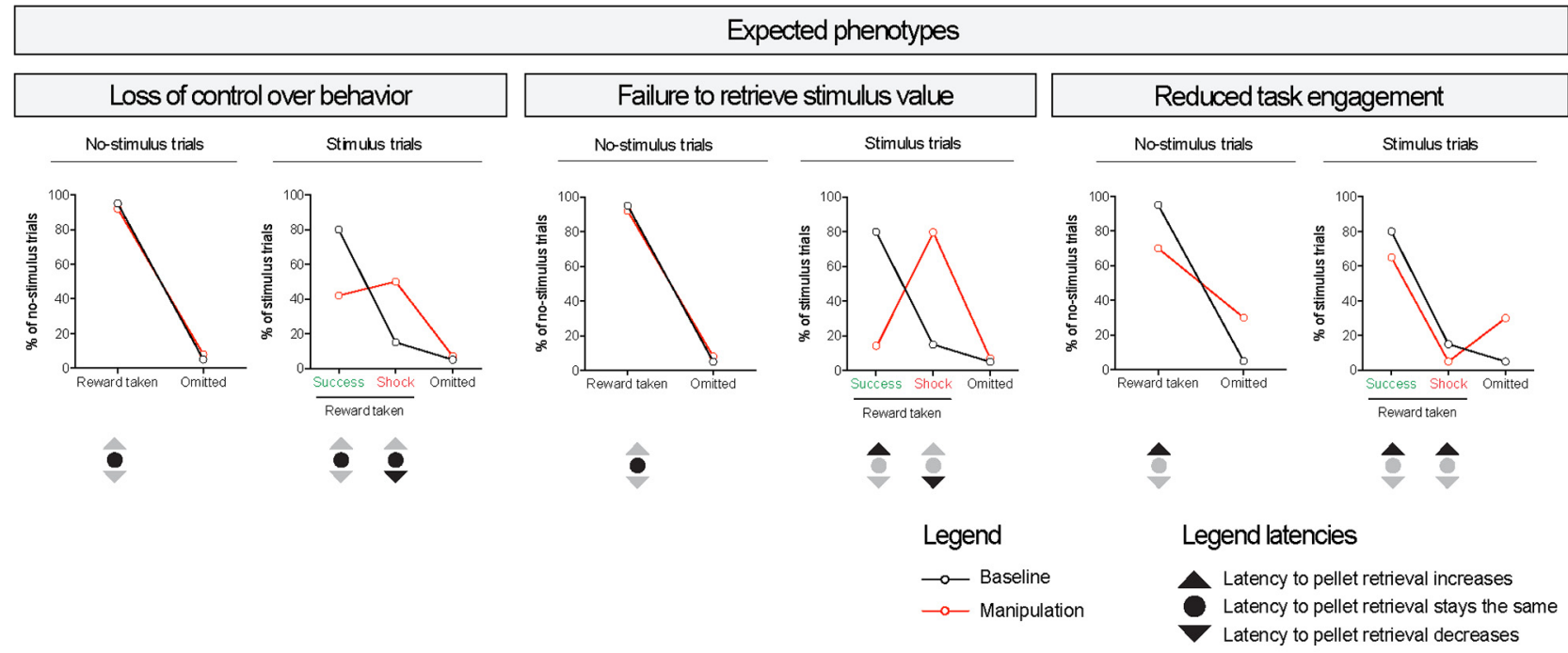

C

Example stimulus trial
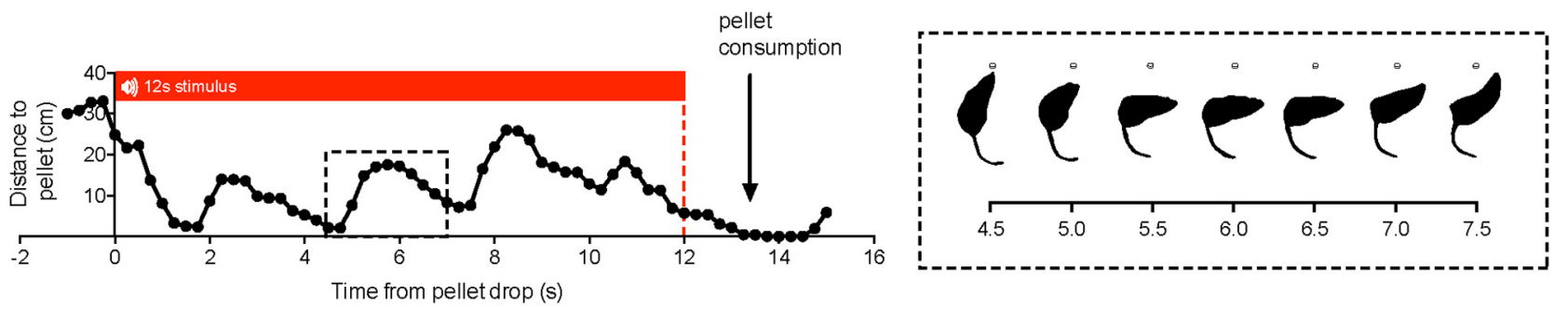

Figure 1. Task description. $\boldsymbol{a}$, Behavioral setup. The task comprised 60 trials in which a sucrose pellet was delivered into a food port. In half of the trials, animals could take the pellet directly without any negative consequences (no-stimulus trials). In the other half of the trials, pellet delivery was accompanied by a $12 \mathrm{~s}$ audiovisual stimulus that signaled to the animals that they had to wait with entering the food port until stimulus termination (stimulus trials). Food port entry during the stimulus was detected by an infrared movement detector and was punished with a $0.3 \mathrm{~s}$ electric foot shock. Inset shows the individual animals' foot shock intensities (median $\pm 25-75$ th percentile, whiskers extend to minimum and maximum values). For training data and latencies per trial type, see Figure 1-1, available at https://doi.org/10.1523/JNEUROSCI.2814-18.2019.f1-1, and Figure 1-2, available at https://doi.org/10.1523/JNEUROSCI.2814-18.2019.f1-2. b, Possible phenotypes after (neural) manipulation. Note that for no-stimulus trials, both options (Reward taken and Omitted) add up to 100\%, as well as for the options during the stimulus trials (Reward taken - Success, Reward taken - Shock, Omitted). Dark arrows under graphs represent possible changes in latency of pellet retrieval for each trial type. For behavioral data of the reduced task engagement phenotype, see Figure 1-3, available at https://doi.org/10.1523/JNEUROSCI.2814-18.2019.f1-3. For statistics, see Figure 1-4, available at https://doi.org/10.1523/JNEUROSCI.281418.2019.f1-4. c, Quantification of a trial from Movie 2, demonstrating attract and repel behavior directed toward and away from the food receptacle during a stimulus trial.

the number of omissions, and thus represents a measure of (loss of) control over behavior.

Expected phenotypes. Based on the trial outcomes and the speed with which animals retrieve the pellets, different behavioral phenotypes can be discerned (Fig. 1b). First, impaired inhibition of behavior, in which animals are not able to refrain from taking the sucrose pellet for the entire stimulus period, would be characterized by an increase in shock trials, at the expense of the number of success trials (Fig. 1b, left). Latency of pellet retrieval during shock trials is likely to be decreased compared with con- trol conditions, i.e., if animals show reduced control over behavior, this may happen earlier in the stimulus period. Behavior during no-stimulus trials should be unchanged, and neither should be the latency of pellet retrieval during success trials (i.e., the speed of food port entry after stimulus offset).

Second, when the animal's capability of retrieving the value of the stimulus is compromised, animals would behave as if there was no threat signal presented at all (Fig. 1b, middle). This would lead to a similar, but more pronounced behavioral pattern as after loss of control over behav- 
ior, and the latency to retrieve pellets will differ. During shock trials, in which the animals take the pellet during the stimulus, retrieval latency will become shorter, as animals are less able to distinguish between nostimulus and stimulus trials. Similarly, latency of food port entry after stimulus offset, during success trials, is likely to be higher, because animals will not successfully process the value of termination of the threat signal. This contrasts with loss of control over behavior, in which tone offset, indicating that it is safe to retrieve the pellet, is processed appropriately, and retrieval latency during success trials will not change. When there is a failure to retrieve stimulus value, the latency of pellet retrieval will occasionally exceed $12 \mathrm{~s}$, regardless of stimulus presentation, which during stimulus trials will be registered as a success trial.

Third, a reduction in task engagement, for example caused by a decreased motivation to obtain reward or by reduced attention, would increase the number of omissions, both in no-stimulus, as well as in stimulus trials (Fig. $1 b$, right). The number of shock trials will likely be low, as it will be easier for the animals to wait with pellet retrieval until after stimulus termination. Furthermore, latency until pellet retrieval is likely to be increased in all trials.

Clearly, behavior could also be disrupted by a combination of these three phenotypes, which would lead to a variety of patterns in trial outcomes and latencies.

Task training. Animals were trained once or twice a day, for 5-7 d per week, starting with magazine training, which was the same task as described in subheading Task except that exclusively no-stimulus trials were presented. Thus, 60 sucrose pellets were delivered into the food port at an interval of $40 \mathrm{~s}$. If the animals made $<5$ omissions in a session, training progressed to the final training phase (Fig. 1-1, available at https://doi.org/10.1523/JNEUROSCI.2814-18.2019.f1-1), which was the task version described in subheading Task.

In the first session of the final training phase, foot shock intensity was set to $0.35 \mathrm{~mA}$. If more than half of the stimulus trials were punished, it was assumed that the intensity was too low to induce effective punishment; hence the foot shock intensity of the next session was increased with 0.05 or $0.1 \mathrm{~mA}$. Similarly, if an animal made many omissions, it was assumed that the foot shock was too intense, and shock intensity was decreased with $0.05 \mathrm{~mA}$ in the next session. After animals reached the criterion of 20 success trials of 30 stimulus trials (meaning that the rat waited with pellet retrieval in $2 / 3$ of the stimulus trials), foot shock intensity was kept constant for the remainder of the experiment. All animals learned the task, so no "non-learners" had to be excluded from the experiment.

Infusions. For the intracranial infusions into the bilateral guide cannulae, double injectors were used that protruded $1 \mathrm{~mm}$ beyond the end of the guides. For the single-guide cannulae, injectors were used that protruded $\sim 0.4 \mathrm{~mm}$ beyond the end of the guide. Animals were habituated to the procedure the day before the experiment, by an infusion of $0.3 \mu \mathrm{l}$ saline through the cannulae.

On testing day, animals received an infusion of a mixture of baclofen (1 nmol; Sigma-Aldrich) and muscimol (0.1 nmol; Sigma-Aldrich) (B/M) dissolved in $0.3 \mu \mathrm{l}$ saline (McFarland and Kalivas, 2001), or $0.3 \mu \mathrm{l}$ saline as a control (counterbalanced between days, $24 \mathrm{~h}$ apart) using a syringe pump (Harvard Apparatus) set at an infusion rate of $0.5 \mu \mathrm{l} / \mathrm{min}$. Thus, animals were tested in the behavioral task twice, according to a counterbalanced, within-subjects design. After infusion, the injectors were kept in place for an additional $30 \mathrm{~s}$ to allow the drug to diffuse into the tissue. After infusion, the dummy cannulae were placed back into the guides, and the animals were returned to their home cage for 10-20 min, before experimental testing commenced.

Free-feeding assay. In the free-feeding assay, animals were infused with baclofen/muscimol or saline, and placed back into their home cage for $2 \mathrm{~h}$. Animals had ad libitum access to chow in a feeding rack that was attached to the wall of the home cage. Food was weighed at the beginning of the experiment and again $2 \mathrm{~h}$ later. The entire protocol was performed twice, once after infusion of baclofen/muscimol and once after infusion of saline (counterbalanced between subjects; $24 \mathrm{~h}$ apart). Thus, testing took place according to a counterbalanced, within-subjects design.

Tail withdrawal test. The tail withdrawal test (Verharen et al., 2018) took place during the $2 \mathrm{~h}$ free-feeding assay (which occurred twice; once

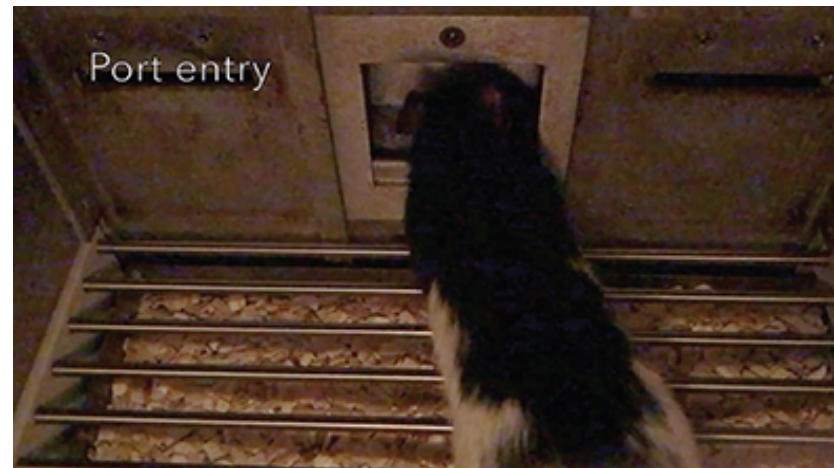

Movie 1. Front view task: all trial types.

after baclofen/muscimol infusion and once after saline infusion, see Free-feeding assay). During this test, the animal was restrained with a towel, and $3-5 \mathrm{~cm}$ of the animal's tail was placed in a beaker containing water of $50 \pm 1^{\circ} \mathrm{C}$. The test was filmed, and latency until tail withdrawal was scored from the movies in a frame-by-frame manner, by a researcher blind to the treatment (baclofen/muscimol or saline).

Histological verification. After the behavioral experiments, animals were transcardially perfused with PBS followed by $4 \%$ paraformaldehyde in PBS. Brains were postfixed in 4\% paraformaldehyde in PBS for $24 \mathrm{~h}$ at $4^{\circ} \mathrm{C}$ followed by a $30 \%$ sucrose solution at $4^{\circ} \mathrm{C}$. Next, brains were cut in coronal slices of $50 \mu \mathrm{m}$ using a cryostat. Brain slices were mounted and colored with 5\% Giemsa (Sigma-Aldrich) dissolved in distilled water. Infusion sites were histologically verified by a researcher blind to the experimental results.

Exclusion criteria. Five animals were excluded based on misplacement of the cannulae: infralimbic cortex, 1 ; anterior cingulate cortex, 1 ; medial orbitofrontal cortex, 1; lateral orbitofrontal cortex, 1; dorsomedial striatum, 1 . Ten animals died during surgery: dorsomedial striatum, 1 ; infralimbic cortex, 2; anterior cingulate cortex, 1; medial orbitofrontal cortex, 2; lateral orbitofrontal cortex, 2; basolateral amygdala, 1; dorsomedial striatum, 1 . One animal was excluded from the basolateral amygdala group because of blockage of the cannula. Infusions into the ventral striatum were initially targeted separately at the nucleus accumbens shell versus core, but were later combined into one ventral striatum group, because the infusion sites were difficult to histologically distinguish. One animal from this group was excluded because it lost its headcap. Data from one animal was removed from the ventral striatum infusion experiment, because the pellet dispenser did not work during the saline session.

Code availability. The MedPC script of the task is available at http:// www.github.com/jeroenphv.

Statistics. Statistical tests were performed with Prism 6.0 (GraphPad Software). Statistical tests were a within-animal (i.e., paired) comparison, in which baclofen/muscimol treatment was compared with saline (baseline) treatment. In these experiments, brain region was not included as a between-subjects factor, because we expected different behavioral phenotypes after inactivation of the different brain regions. In the free-feeding assay and tail withdrawal test, a two-way repeated-measure ANOVA was used, with baclofen/muscimol versus saline as a withinsubjects repeated measures factor, and treatment group (brain area) as a between-subjects factor. In all figures, statistical significance is denoted with the following range: ${ }^{\#} p<0.1,{ }^{*} p<0.05,{ }^{* *} p<0.01,{ }^{* * *} p<$ $0.001,{ }^{* * * *} p<0.0001$. Unless otherwise stated, all bar graphs indicate the mean with standard error of the mean. Extended statistics are presented Fig. 1-4, available at https://doi.org/10.1523/JNEUROSCI.2814-18. 2019.f1-4. 


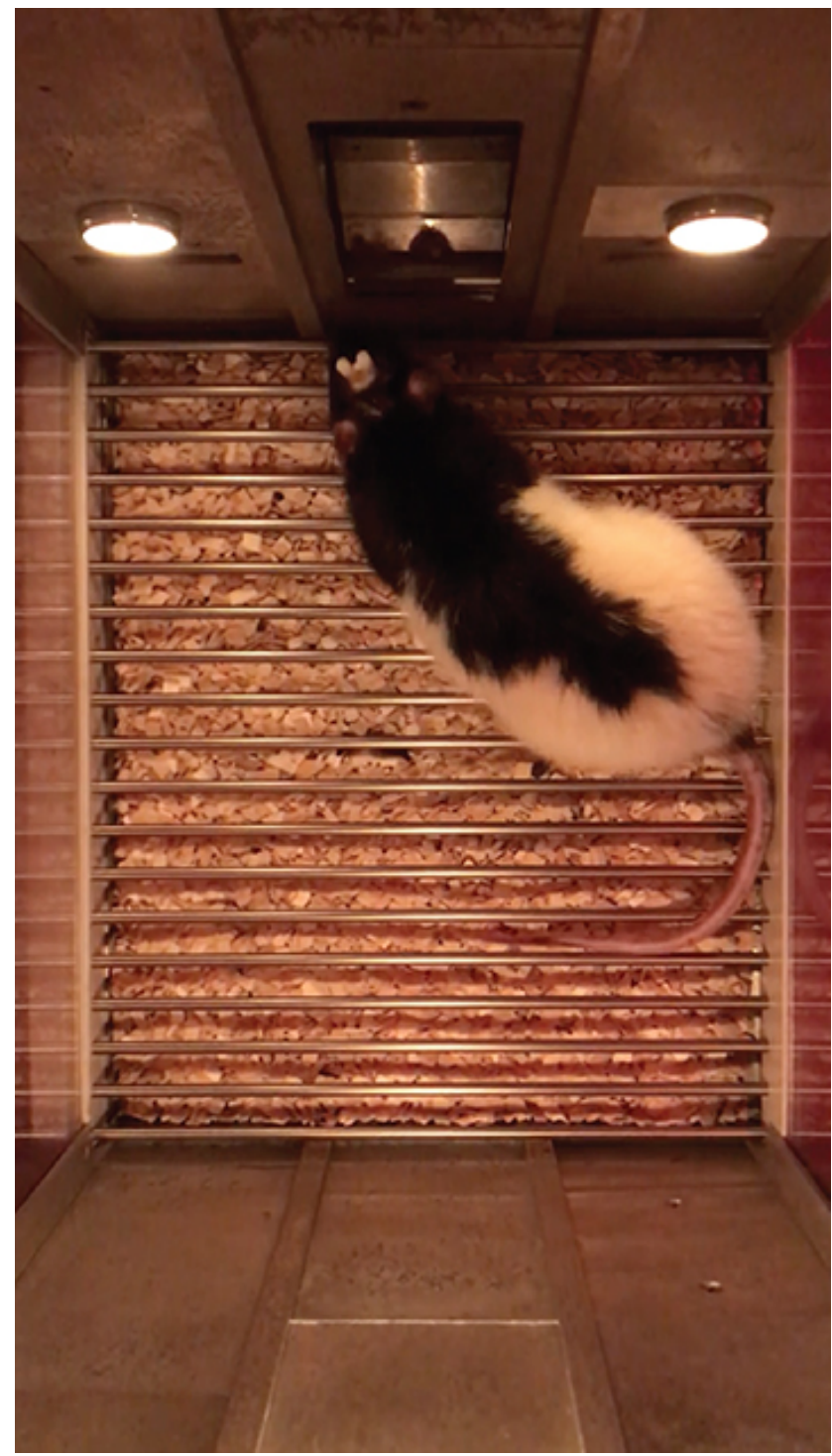

Movie 2. Top view task: two trials of the stimulus-success type.

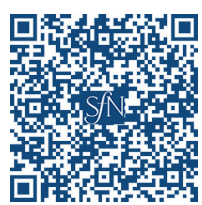

\section{Results}

Task behavior

All rats learned the task, i.e., they managed to wait to eat the pellet during the stimulus in the majority of trials (Movies 1,2 ). In success trials, the rats retrieved the pellet quickly after tone offset, with an average latency of $\sim 2.5 \mathrm{~s}$ (Fig. 1-2, available at https:// doi.org/10.1523/JNEUROSCI.2814-18.2019.f1-2). In shock trials, i.e., trials in which animals retrieved the pellet during the stimulus and hence received punishment, latencies of pellet retrieval were usually higher than in no-stimulus trials ( $\sim 5 \mathrm{~s}$ compared with $\sim 1.5 \mathrm{~s}$ in no-stimulus trials; Fig. 1-2, available at https://doi.org/10.1523/JNEUROSCI.2814-18.2019.f1-2), as if the rats managed to control their behavior for a fraction of the stimulus period before they reached out for the pellet. Interestingly, animals typically exhibited "attract and repel" behavior directed toward and away from the food receptacle during behavioral control (Fig. 1c; Movies 1,2).

\section{Pre-feeding devaluation evokes a "reduced task} engagement" phenotype

As described in Materials and methods, different phenotypes can be discerned on the basis of the trial outcomes and the speed with which animals retrieve the pellet (Fig. $1 b$ ). As proof-of-principle, we pre-fed a group of animals with sucrose pellets before the task, to evoke a phenotype of reduced task engagement, by decreasing the motivation to obtain sucrose reward. Indeed, this induced a pattern of effects (Fig. 1-3, available at https://doi.org/10.1523/ JNEUROSCI.2814-18.2019.f1-3) that matched expectations (Fig. $1 b$, right). That is, the number of omissions increased, animals showed increased control over behavior, and there was a trend toward increased latencies of pellet retrieval during no-stimulus trials and success trials. No effect was observed on the latency of pellet retrieval in shock trials, but note that this latency was only based on data from six animals after pre-feeding, as the other six animals never retrieved the pellet during the stimulus.

\section{Medial PFC inactivation impairs inhibition over behavior}

To study the involvement of different regions of the corticolimbic system to behavior in our task, we pharmacologically inactivated different regions of this system by means of intracranial infusions of the GABA receptor agonists baclofen and muscimol (McFarland and Kalivas, 2001). Inactivation of the prelimbic cortex significantly increased the number of shock trials, which came at the expense of the number of success trials, without affecting behavior during nostimulus trials, or the number of omissions (Fig. 2a). Consequently, the shock index, which measures the fraction of stimulus trials in which the animal retrieved the pellet during the stimulus, thus receiving foot shock, increased significantly. No significant effects on the speed with which the animals retrieved the pellet were observed (Fig. 2a). This pattern of effects matches the phenotype corresponding to loss of control over behavior (Fig. 1b), suggesting that inactivation of the prelimbic cortex impaired the ability of animals to inhibit their urge to approach the pellet, despite the presence of the threat signal. Inactivation of the infralimbic cortex yielded the same pattern of effects (Fig. 2b). That is, an increase in the number of shock trials, a decrease in success trials and an increased shock index, without a change in behavior during no-stimulus trials or an effect on any of the latency measures.

Medial orbitofrontal cortex inactivation also impaired control over behavior, as apparent by a significant increase in the number of shock (but not a decrease in success) trials and thereby an increase in the shock index, although this effect was numerically smaller than after inactivation of the prelimbic and infralimbic cortices (Fig. 2c). In addition, it significantly decreased the latency of pellet retrieval during shock trials, indicating that if the animals entered the food port during the stimulus, this happened during an earlier stage of stimulus presentation. This suggests that if the animals lost control over behavior after medial orbitofrontal cortex inactivation, they were able to inhibit themselves for a shorter period of time compared with baseline.

Inactivation of the anterior cingulate cortex also evoked disinhibition of behavior. Thus, the number of success trials was reduced, and the number of shock trials, as well as the shock index, increased. Latency analysis revealed that animals also became slower in pellet retrieval during no-stimulus trials, where they were allowed to consume the pellet directly without negative consequences (Fig. 2d). Thus, animals responded slower than under baseline conditions, suggesting that additional cognitive functions, such as attention, could be impaired.

Together, these data suggest that these four medial prefrontal regions have an important role in mediating control over behav- 
a

b

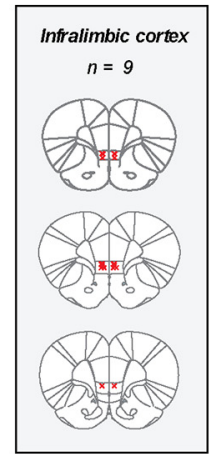

C

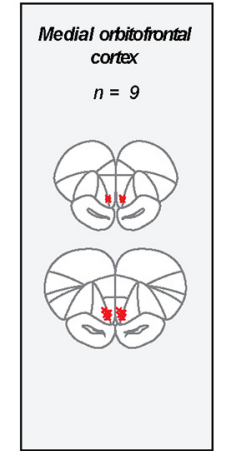

d

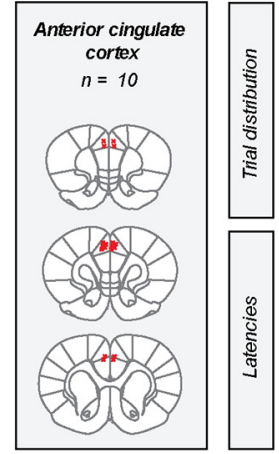

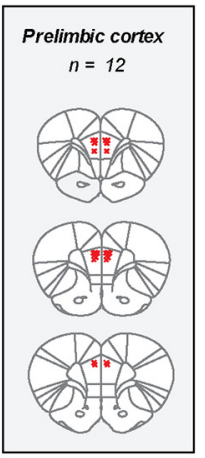
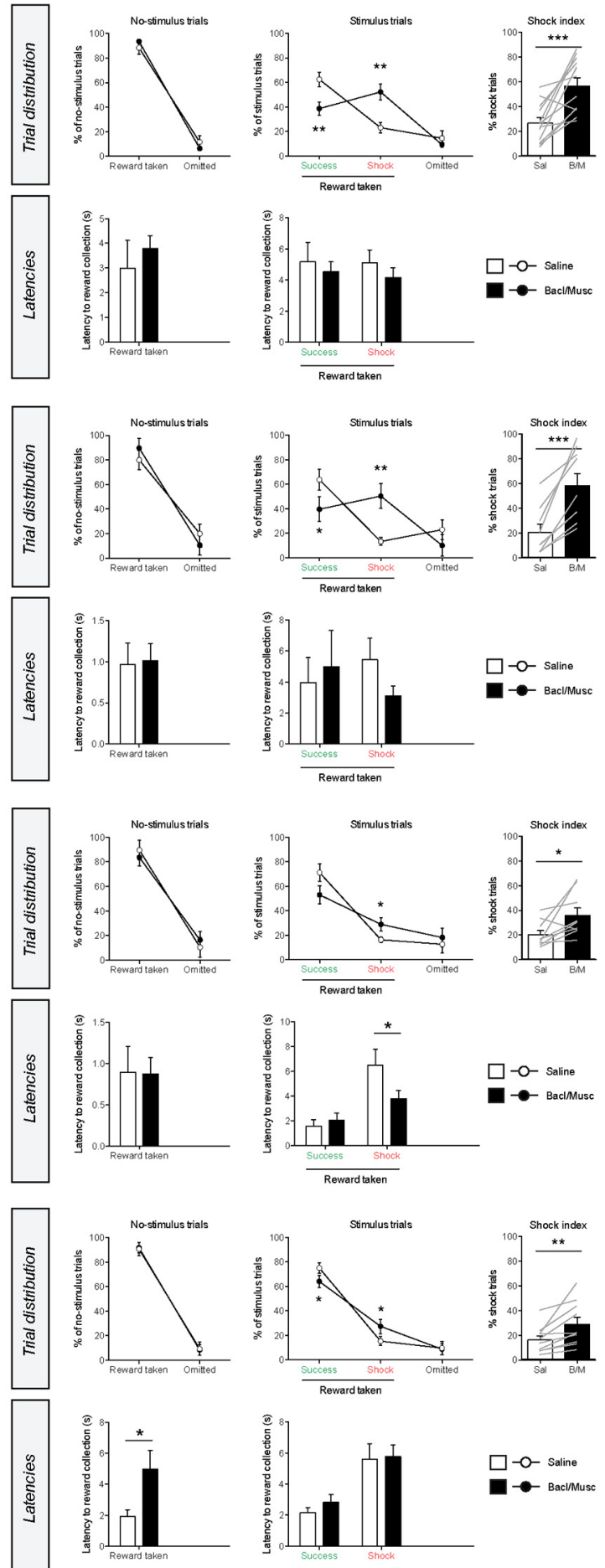
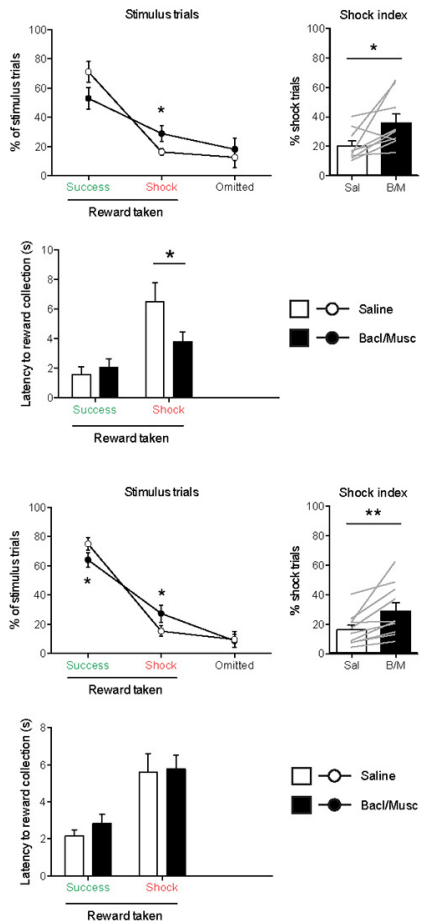

Figure 2. Effects of pharmacological inactivation of the medial PFC on task behavior. $\boldsymbol{a}$, Effects of prelimbic cortex inactivation. No-stimulus trials, reward taken: paired $t$ test, $t_{(11)}=1.1, p=0.30$; latency: $t_{(11)}=0.6, p=0.55$. Stimulus trials, number of success trials: $t_{(11)}=3.2, p=0.0079$, latency of success trials: $t_{(11)}=0.5, p=0.66$; number of shock trials: $t_{(11)}=4.2, p=$ 0.0015 , latency of shock trials: $t_{(11)}=0.8, p=0.42$; number of omissions: $t_{(11)}=1.0, p=0.35$; Shock index: $t_{(11)}=4.5, p=$ 0.0008 . $\boldsymbol{b}$, Effects of infralimbic cortex inactivation. No-stimulus trials, reward taken: paired $t$ test, $t_{(8)}=1.7, p=0.12$; latency: $t_{(8)}$ $=0.2, p=0.83$. Stimulus trials, number of success trials: $t_{(8)}=2.6, p=0.0293$, latency of success trials: $t_{(8)}=0.7, p=0.51_{\text {; }}$ number of shock trials: $t_{(8)}=4.5, p=0.0021$, latency of shock trials: $t_{(8)}=1.8, p=0.11$; number of omissions: $t_{(8)}=2.0, p=$ 0.084 . Shock index: $t_{(8)}=5.8, p=0.0004$. c, Effects of medial orbitofrontal cortex inactivation. No-stimulus trials, reward taken: paired $t$ test, $t_{(8)}=0.5, p=0.61$; latency: $t_{(8)}=0.1, p=0.94$. Stimulus trials, number of success trials: $t_{(8)}=2.0, p=0.0840$, latency of success trials: $t_{(8)}=0.8, p=0.47$; number of shock trials: $t_{(8)}=2.5, p=0.0348$, latency of shock trials: $t_{(8)}=2.5, p=$ 0.0360; number of omissions: $t_{(8)}=0.6, p=0.59$. Shock index: $t_{(8)}=0.3, p=0.0316$. $\boldsymbol{d}$, Effects of anterior cingulate cortex inactivation. No-stimulus trials, reward taken: paired $t$ test, $t_{(9)}=0.5, p=0.66$; latency: $t_{(9)}=0.3, p=0.0262$. Stimulus trials, number of success trials: $t_{(9)}=2.7, p=0.0235$, latency of success trials: $t_{(9)}=0.1, p=0.23$; number of shock trials: $t_{(9)}=3.2$, $p=0.0114$, latency of shock trials: $t_{(9)}=0.2, p=0.88$; number of omissions: $t_{(9)}=0.6, p=0.56$. Shock index: $t_{(9)}=0.3$, ior in this task, whereby the prelimbic and infralimbic function is most criti$\mathrm{cal}$, and the anterior cingulate cortex may also serve other cognitive functions that are necessary for correct task execution.

\section{Lateral orbitofrontal cortex and basolateral amygdala inactivation disrupt task performance}

Inactivation of the lateral orbitofrontal cortex and the basolateral amygdala impaired task performance, but in different ways. After inactivation of the lateral orbitofrontal cortex, we observed a significant increase in the number of omitted trials, as well as higher latencies of pellet retrieval in no-stimulus trials and success trials (Fig. 3a). Furthermore, no decrease in the number of shock trials was observed, hence the shock index was significantly increased. This pattern of effects suggests a reduction in task engagement.

Basolateral amygdala inactivation induced a dramatic increase in the number of shock trials, leading to a shock index of $\sim 90 \%$, meaning that 9 of 10 port entries during stimulus trials were during the stimulus, and hence were punished (Fig. $3 b$ ). No effect was observed on the number of omissions during stimulus trials, and neither were there any effects on behavior during no-stimulus trials. Thus, basolateral amygdala inactivation only affected behavior around the stimulus presentation. Interestingly, the latency of pellet retrieval during shock trials was reduced (i.e., animals were able to control their behavior for a shorter amount of time), whereas an increase in latency was observed during success trials (i.e., after successful control, animals did not directly take the pellet after stimulus offset). This pattern of effects (Fig. 1b) suggests that basolateral amygdala inactivation impaired the ability of the animals to retrieve the value of the stimulus, so that animals ostensibly behaved as if there was no threat signal presented. As such, certain

$p=0.0085$. The shock index is the number of shock trials as a fraction of the stimulus trials in which reward was taken. Red crosses in the coronal brain sections represent the infusion sites in each experiment. Gray lines in shock index graphs indicate individual animals. For latency analyses, the median latency per animal per trial type was used. Latency in success trials represents the latency to pellet retrieval after stimulus offset; in other trials the latencies represent the latency of pellet retrieval after reward delivery. ${ }^{*} p<0.05,{ }^{* *} p<0.01$, ${ }^{* * *} p<0.001$ in paired $t$ test (for statistical table, see Figure 1-4, available at https://doi.org/10.1523/JNEUROSCI.281418.2019.f1-4). 
a
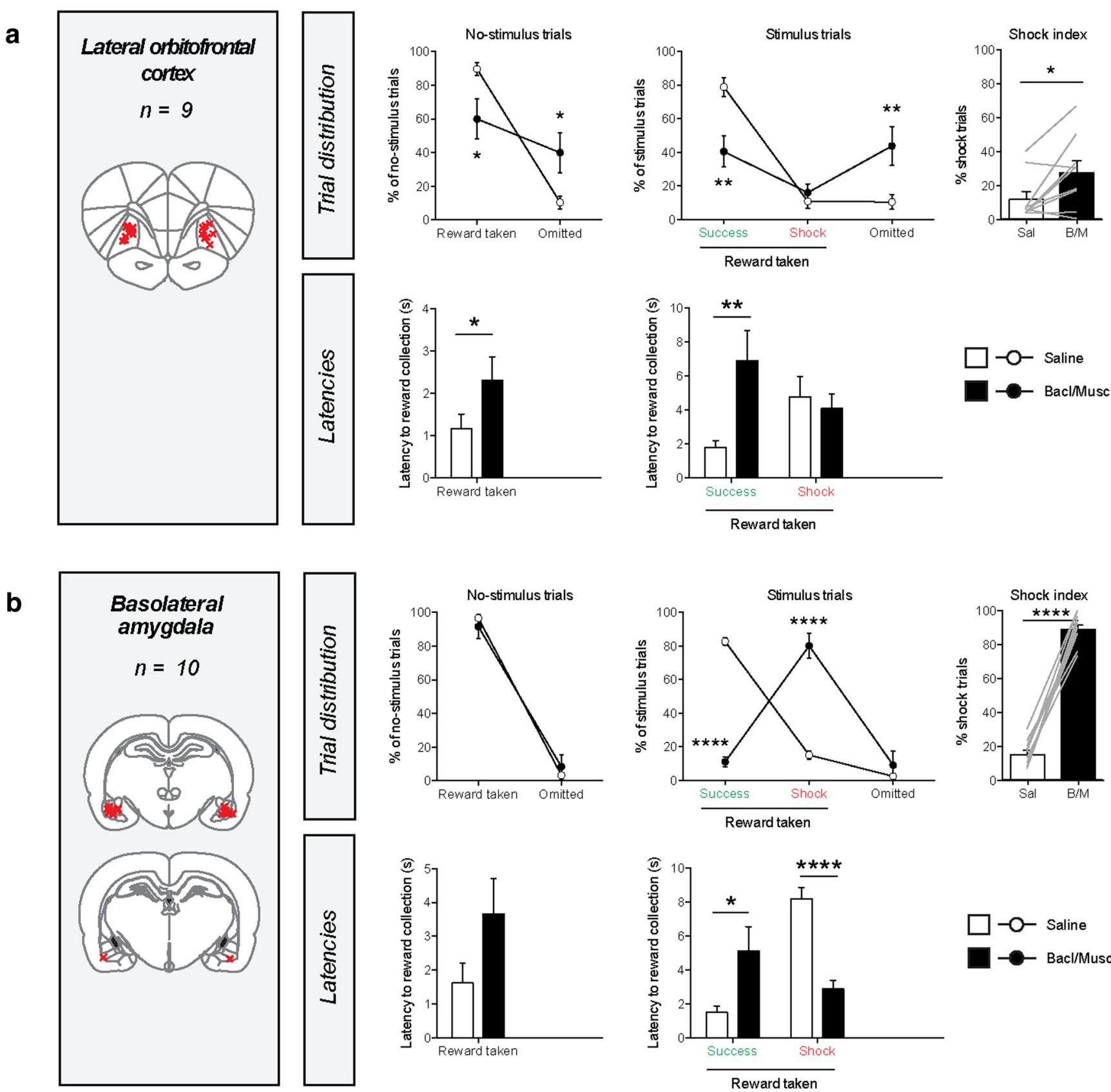

Figure 3. Effects of pharmacological inactivation of the lateral orbitofrontal cortex and basolateral amygdala on task behavior. $\boldsymbol{a}$, Effects of lateral orbitofrontal cortex inactivation. No-stimulus trials, reward taken: paired $t$ test, $t_{(8)}=3.1, p=0.0157$; latency: $t_{(7)}=0.3, p=0.0286$. Stimulus trials, number of success trials: $t_{(8)}=4.8, p=0.0014$, latency of success trials: $t_{(8)}=3.4, p=0.0091$; number of shock trials: $t_{(8)}=1.0, p=0.35$, latency of shock trials: $t_{(7)}=0.01, p=0.99 ;$ number of omissions: $t_{(8)}=0.4, p=0.0050$. Shock index: $t_{(8)}$ $=2.8, p=0.0235 . \boldsymbol{b}$, Effects of basolateral amygdala inactivation. No-stimulus trials, reward taken: paired $t$ test, $\left.t_{(9)}=0.6, p=0.53\right)$; latency: $t_{(9)}=1.6, p=0.14$. Stimulus trials, number of success trials: $t_{(9)}=21.2, p<0.0001$, latency of success trials: $t_{(7)}=2.7, p=0.0317$; number of shock trials: $t_{(9)}=8.0, p<0.0001$, latency of shock trials: $t_{(9)}=8.3, p<$ 0.0001 ; number of omissions: $t_{(9)}=0.8, p=0.46$. Shock index: $t_{(9)}=19.3, p<0.0001$. For an additional latency analysis of this experiment, see Figure 3-1, available at https://doi.org/10.1523/JNEUROSCI.2814-18.2019.f3-1. The shock index is the number of shock trials as a fraction of the stimulus trials in which reward was taken. Red crosses in the coronal brain sections represent the infusion sites in each experiment. Gray lines in shock index graphs indicate individual animals. Latency in success trials represents the latency to pellet retrieval after stimulus offset; in other trials the latencies represent the latency of pellet retrieval after reward delivery. ${ }^{*} p<0.05,{ }^{* *} p<0.01,{ }^{* * *} p<0.001,{ }^{* * * *} p<0.0001$ in paired $t$ test (for statistical table, see Figure 1-4, available at https://doi.org/10.1523/JNEUROSCI.2814-18.2019.f1-4).

stimulus trials could be counted as a success trial merely because the animals were sometimes slow in retrieving the pellet (regardless of stimulus presentation). If this were true, then the latency of pellet retrieval during no-stimulus trials would also occasionally exceed the $12 \mathrm{~s}$ threshold. To test this notion, we analyzed the latencies of no-stimulus trials after baclofen/ muscimol infusion, and observed that on average $15.7 \pm 3.7 \%$ of no-stimulus trials had a pellet retrieval latency of $12 \mathrm{~s}$ or more; this was statistically indistinguishable from the fraction of stimulus trials that was counted as a success trial, which was $11.0 \pm 2.9 \%$ of trials (Fig. $3-1 a$, available at https://doi.org/ 10.1523/JNEUROSCI.2814-18.2019.f3-1). Furthermore, the distribution of latencies did not differ between no-stimulus trials and stimulus trials (Fig. 3-1b, available at https:// doi.org/10.1523/JNEUROSCI.2814-18.2019.f3-1). Together, this shows that on the basis of the conventional task parame- 
a
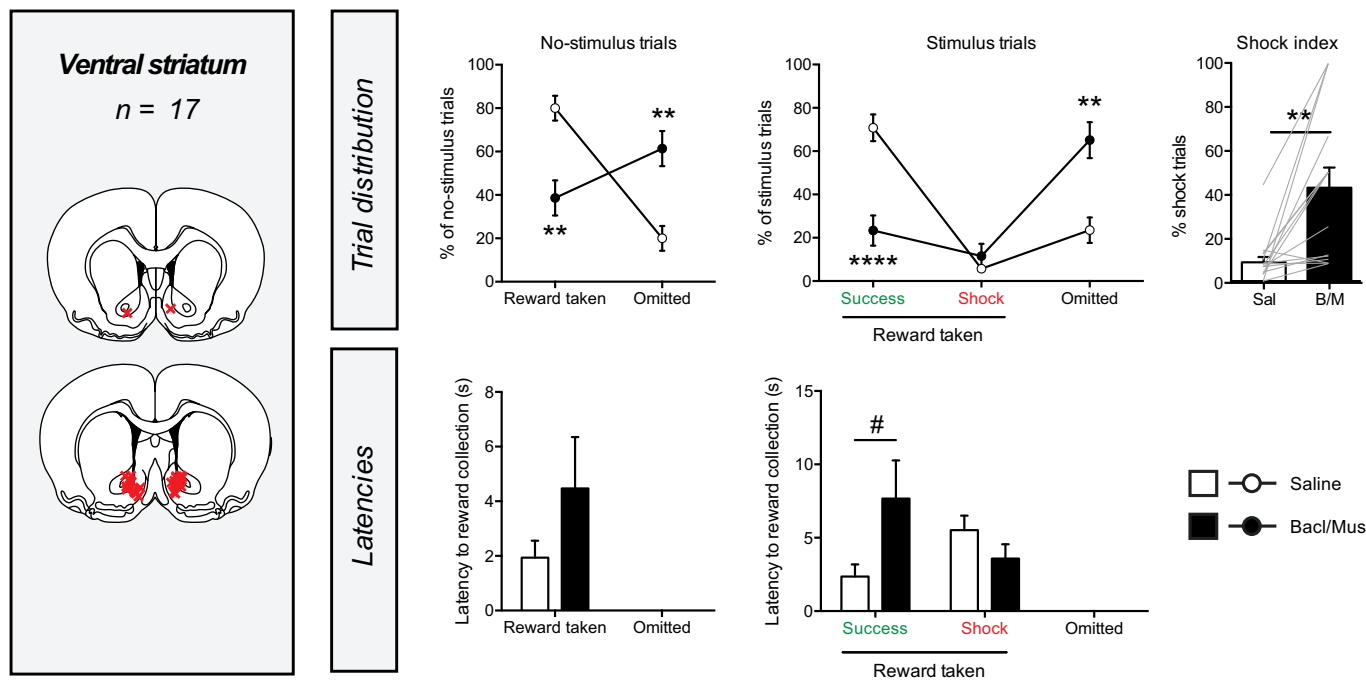

b
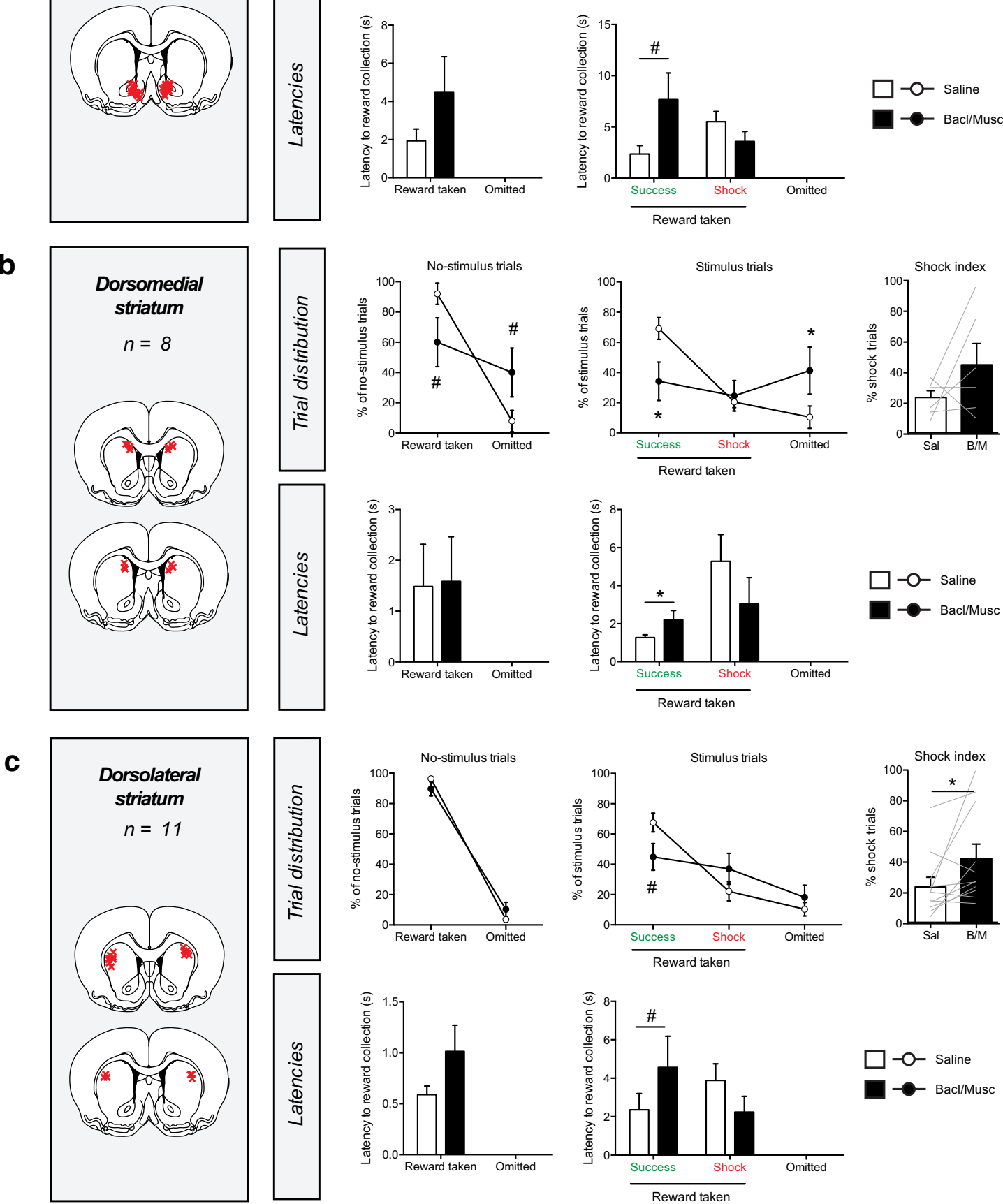

Figure 4. Effects of pharmacological inactivation of striatal subregions on task behavior. $\boldsymbol{a}$, Effects of ventral striatum inactivation. No-stimulus trials, reward taken: paired $t$ test, $t_{(16)}=3.9, p=$ 0.0013 ); latency: $t_{(14)}=1.1, p=0.28$. Stimulus trials, number of success trials: $t_{(16)}=5.5, p<0.0001$, latency of success trials: $t_{(11)}=2.1, p=0.064 ;$; number of shock trials: $t_{(16)}=1.1, p=$ 0.28 , latency of shock trials: $t_{(12)}=1.2, p=0.24$; number of omissions: $t_{(16)}=3.7, p=0.0020$. Shock index: $t_{(14)}=4.0, p=0.0014$. For the animals for which the cannulas ended up exclusively in the nucleus accumbens shell, see Figure 4-1, available at https://doi.org/10.1523/JNEUROSCI.2814-18.2019.f4-1. $\boldsymbol{b}$, Effects of dorsomedial striatum inactivation. No-stimulus trials, reward taken: paired $t$ test, $t_{(7)}=2.3, p=0.056$; latency: $t_{(5)}=1.2, p=0.30$. Stimulus trials, number of success trials: $t_{(7)}=2.6, p=0.0337$, latency of success trials: $t_{(5)}=2.6, p=0.0462 ;$ number of shock trials: $t_{(7)}=0.4, p=0.67$, latency of shock trials: $t_{(5)}=1.3, p=0.26$; number of omissions: $t_{(7)}=2.5, p=0.0421$. Shock index: $t_{(7)}=1.5, p=0.21$. $c$, Effects of dorsolateral striatum inactivation. No-stimulus trials, reward taken: paired $t$ test, $t_{(10)}=1.3, p=0.22$; latency: $t_{(10)}=1.7, p=0.13$. Stimulus trials, number of success trials: $t_{(10)}=2.1, p=0.057$, latency of success trials: $t_{(9)}=$ $1.9, p=0.095$; number of shock trials: $t_{(10)}=1.8, p=0.10$, latency of shock trials: $t_{(10)}=1.3, p=0.22$; number of omissions: $t_{(10)}=0.9, p=0.38$. Shock index: $t_{(10)}=2.2, p=0.0486$. The shock index is the number of shock trials as a fraction of the stimulus trials in which reward was taken. Red crosses in the coronal brain sections represent (Figure legend continues.) 
ters, behavior between no-stimulus trials and stimulus trials could not be distinguished, suggesting that after basolateral amygdala inactivation, animals behaved as if they could not retrieve the value of the threat stimulus.

\section{Activity in the striatum is important for task engagement}

After pharmacological inactivation of the ventral striatum, we observed a significant increase in the number of omissions during both trial types (Fig. 4a). During stimulus trials, this occurred at the expense of the number of success trials. No change in the number of shock trials was observed. Because of this decrease in the number of success trials, we observed an increase in the shock index, as the relative amount of shock trials increased. Although no effects on latencies of pellet retrieval were found, it must be noted that because of the large amount of omissions, these latencies were based on a lower number of trials (or even no trials for animals that exclusively made omissions). After histological verification of the infusion sites, we observed that most guide cannulae were positioned above the core region of the nucleus accumbens. However, when only analyzing the animals in which the infusions were targeted at the nucleus accumbens shell, a comparable pattern of effects was observed (Fig. 4-1, available at https://doi.org/10.1523/JNEUROSCI.2814-18.2019.f4-1).

Pharmacological inactivation of the dorsomedial striatum resulted in a similar, although more modest pattern of effects. As such, infusion of baclofen and muscimol resulted in a trend toward an increase in the number of omissions during no-stimulus trials and a significant increase in omissions during stimulus trials, which was associated with a reduction in the number of success trials (Fig. 3b). However, no significant increase in the shock index was observed. Similar to the phenotype observed after ventral striatum inactivation, a significant increase in the latency of pellet retrieval was observed during success trials, with no changes in the latencies in shock trials or in no-stimulus trials.

Pharmacological inactivation of the dorsolateral striatum induced a pattern of effects that somewhat resembled the effects seen after ventral striatum inactivation, in that a trend toward a decrease in the number of success trials was observed, a trend toward an increase in latency of pellet retrieval in these trials, and a significant increase in the shock index (Fig. 3c). Critically, however, no effects were observed on the number of omitted trials, neither in no-stimulus nor in stimulus trials, which suggests a strongly attenuated variant of the reduced task engagement phenotype, or perhaps a different behavioral phenotype than after ventral striatum infusion. Similar to ventral striatum inactivation, no changes in latencies were observed in no-stimulus trials or in shock trials.

\section{Control experiments}

As a negative control region, we inactivated the dorsolateral part of the olfactory cortex, which is located ventral of the lateral orbitofrontal cortex (Fig. 5a). This inactivation did not affect any of the behavioral parameters, indicating that the olfactory cortex is not essential for task performance, and it suggests that the

\section{$\leftarrow$}

(Figure legend continued.) the infusion sites in each experiment. Gray lines in shock index graphs indicate individual animals. Latency in success trials represents the latency to pellet retrieval after stimulus offset; in other trials the latencies represent the latency of pellet retrieval after reward delivery. ${ }^{* * *} p<0.0001,{ }^{* * *} p<0.001,{ }^{* *} p<0.01,{ }^{*} p<0.05$, $\# p<0.1$ in paired $t$ test (for statistical table, see Figure 1-4, available at https://doi.org/10.1523/ JNEUROSCI.2814-18.2019.f1-4). infused GABA receptor agonists did not spread throughout the brain to induce infusion site-unspecific behavioral effects.

It is possible that changes in nociception underlie the effects we observed in this study. For example, animals may become less or more sensitive to the foot shock, resulting in changes in behavior during stimulus trials. To control for this possible effect, we performed a tail withdrawal test in the animals, and observed no changes in latency to tail withdrawal after inactivation of the brain regions in which we found increases in the number of shock trials (Fig. 5b). We also conducted a free-feeding assay, because changes in appetite may change behavior in tasks that involve food reward (Fig. 1-3, available at https://doi.org/10.1523/ JNEUROSCI.2814-18.2019.f1-3). In none of the brain areas we observed changes in chow intake in the $2 \mathrm{~h}$ following baclofen/ muscimol infusion (Fig. $5 c$ ). These findings suggest that the observed effects in the behavioral task were not induced by changes in nociception or hunger.

\section{Discussion}

In this study, we presented a novel task that studies the ability of rats to inhibit their urge to consume a visibly present food reward during the presentation of an audiovisual threat signal. Importantly, in this task, control over behavior comprises refraining from consumption of palatable food. Given that many day-today examples of loss of control over behavior encompass behavior directed at primary rewards, like food or drugs, this task aims to provide a more naturalistic approach to inhibition of behavior, because the animals have to balance two innate urges: approach to food reward versus an avoidance response to punishment. An additional benefit is that this task requires relatively little training (Fig. 1-1, available at https://doi.org/10.1523/JNEUROSCI.281418.2019.f1-1), as animals do not have to learn any operant responses to receive reward. Furthermore, using this task, we can discern impairments in different aspects of task performance, including a failure to retrieve stimulus value, a reduction in task engagement, and compromised inhibition of behavior. One drawback of our task is that once animals have acquired the task, they often have very low baseline levels of shock trials, making strengthening of behavioral control hard to detect.

Our behavioral inhibition paradigm shows similarities to certain models of relapse to drug seeking, in which animals are confronted with behavioral conflict between pursuing (drug) reward and avoiding punishment (Cooper et al., 2007; Marchant et al., 2013), based on the classic "obstruction box" setup (Jenkins et al., 1926). However, there are important differences between our approach and conventional tasks for impulse control, such as the 5-choice serial reaction time task (5CSRTT; Carli et al., 1983; Robbins, 2002), the delayed reward task (Evenden and Ryan, 1996), and the stop-signal task (Eagle and Robbins, 2003). First, in our task, impaired inhibition of behavior is punished with foot shock, rather than non-presentation of food. As a result, during the execution of stimulus trials, animals typically exhibit attract and repel behavior toward and away from the food receptacle (Fig. 1c), which is likely the result of an inner conflict between pursuing reward and avoiding punishment. This indicates that the behavior modeled in our task is mechanistically different from the 5CSRTT and the stop-signal task, in which avoidance of punishment is not a factor in guiding behavior, and the neural substrates of punishment (Jean-Richard-Dit-Bressel et al., 2018) are therefore not involved. In contrast, the task presented here involves explicit punishment in the form of electric foot shock. As such, consuming the pellet during stimulus presentation can actually be regarded as suboptimal decision making, as it will not 
a
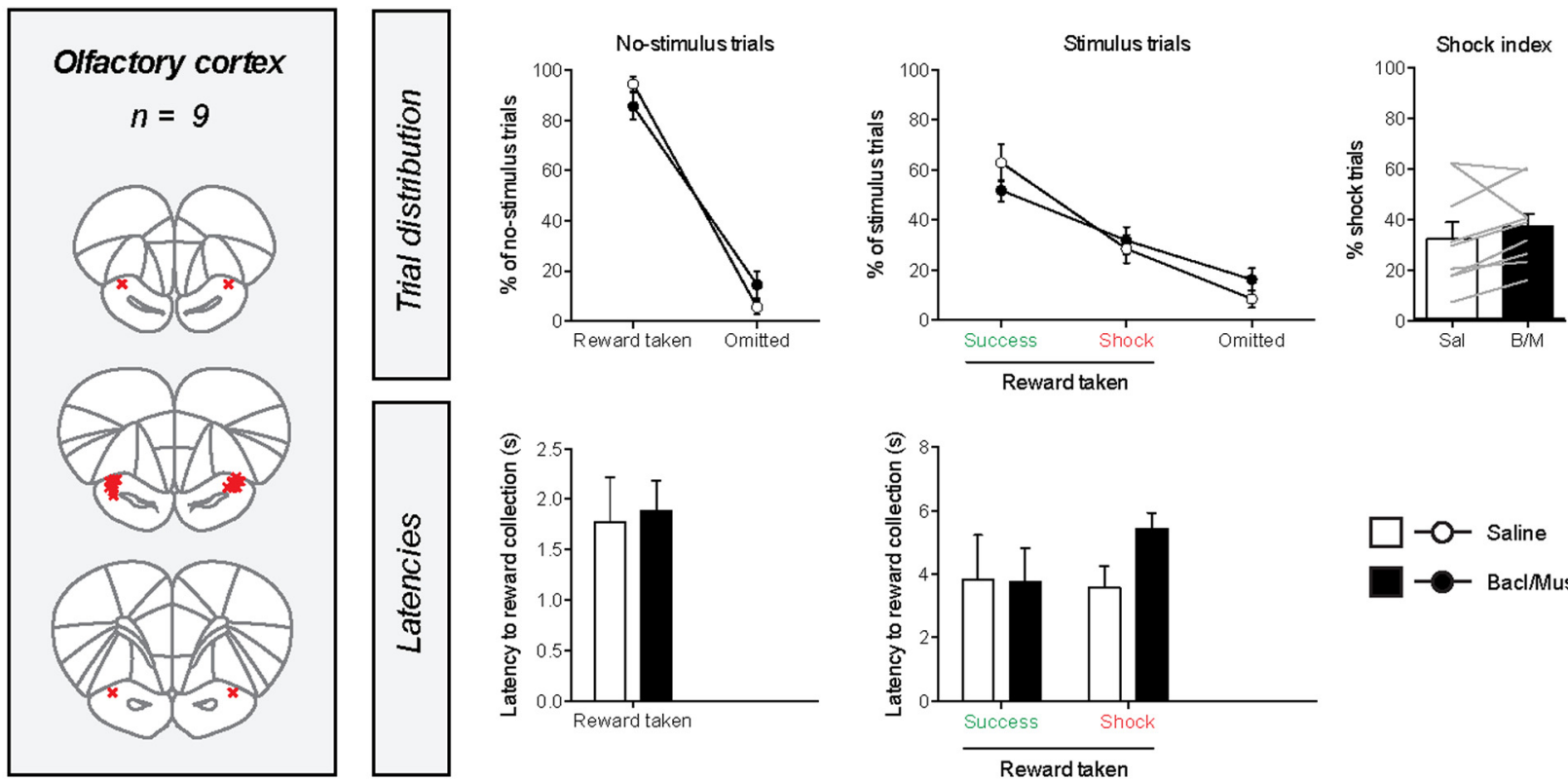

b

tail withdrawal test

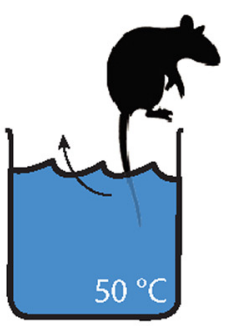

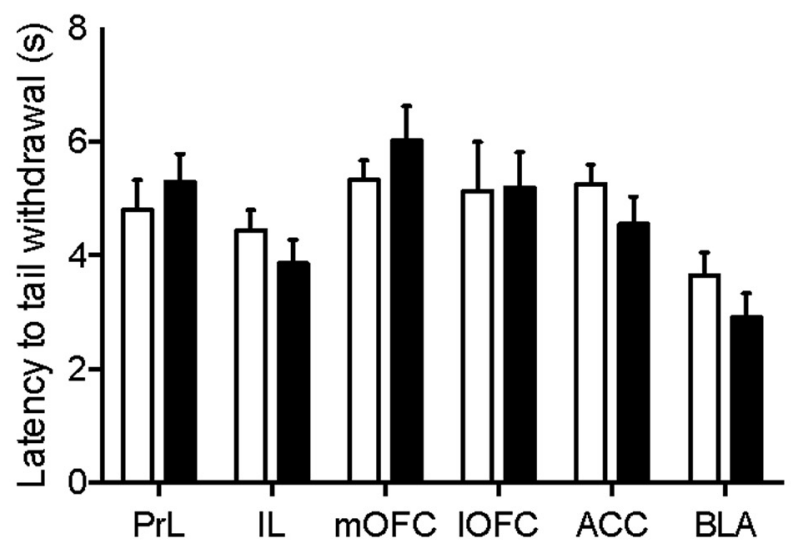

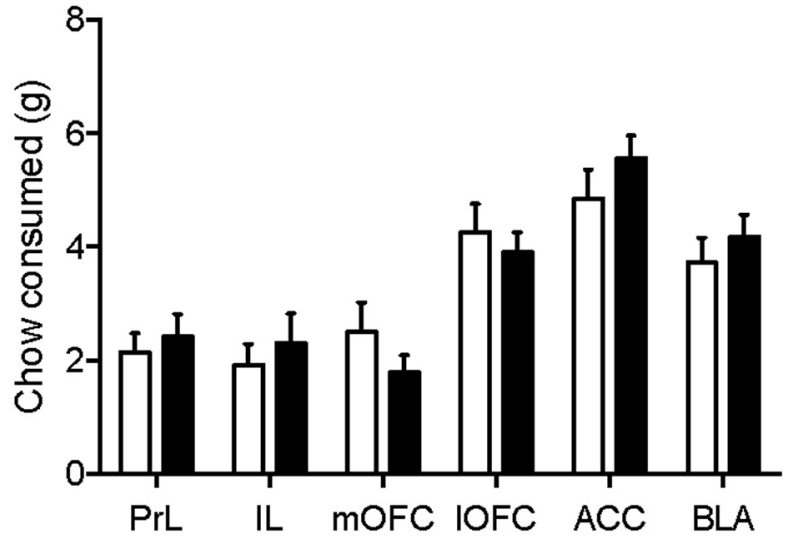

PrL $\square$ Saline

- Baclofen/Muscimol
C

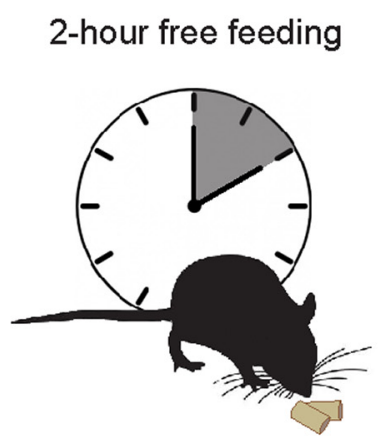

L

Figure 5. Control experiments. $\boldsymbol{a}$, Effects of pharmacological inactivation of the olfactory cortex on task behavior. No-stimulus trials, reward taken: paired $t$ test, $t_{(8)}=1.4, p=0.21 ;$ latency: $t_{(8)}=0.2, p=0.83$. Stimulus trials, number of success trials: $t_{(8)}=1.8, p=0.12$, latency of success trials: $t_{(8)}=0.1, p=0.95 ;$ number of shock trials: $t_{(8)}=1.3, p=0.25$, latency of shock trials: $t_{(8)}=2.1, p=0.066$; number of omissions: $t_{(8)}=1.3, p=0.25$. Shock index: $t_{(8)}=1.3, p=0.23$. $\boldsymbol{b}$, Pharmacological inactivations did not the change latency to tail withdrawal in a tail withdrawal test $\left(n=59\right.$ rats, two-way repeated-measures ANOVA, no main effect of infusion: $F_{(1,53)}=0.33, p=0.57$; or infusion $\times$ group interaction effect: $F_{(5,53)}=1.29, p=0.28$ ). $c$, Pharmacological inactivations did not change chow intake in a free-feeding assay (two-way repeated-measures ANOVA, no main effect of infusion: $n=59$ rats, $F_{(1,53)}=0.35, p=0.55$; or infusion $\times$ group interaction effect: $\left.F_{(5,53)}=1.02, p=0.41\right)$. For statistical table, see Figure 1-4, available at https://doi.org/10.1523/ JNEUROSCI.2814-18.2019.f1-4. PrL, prelimbic cortex; IL, infralimbic cortex; mOFC, medial orbitofrontal cortex; IOFC, lateral orbitofrontal cortex; ACC, anterior cingulate cortex; BLA, basolateral amygdala. 
affect the total reward that can be obtained by the animal. However, taking reward during the stimulus might in some cases reflect the outcome of a cost/benefit decision, in which the foot shock outweighed the value of the temporally discounted reward. In recent years, behavioral tasks have been developed that study whether rats are willing to endure foot shock punishment in exchange for a large sucrose reward (Simon et al., 2009), which was shown to rely on functional activity in the basolateral amygdala and lateral orbitofrontal cortex (Orsini et al., 2015). In addition, the actual presence and mere sight of the food reward makes this task different from the tasks mentioned above, in which behavioral control is expressed through appropriate responding on manipulanda such as levers or nose-poke holes, and the food reward is only presented after expression of correct behavior. As such, in these tasks, levers and nose-poke holes can be viewed as discriminative stimuli, in which control over behavior may rely on other cognitive processes than direct control over palatable food intake (cf. Ambroggi et al., 2011).

Utilizing our new paradigm, we found that a wide array of corticolimbic regions is involved in the proper exertion of behavioral inhibition. Inactivation of the ventral parts of the medial PFC (prelimbic, infralimbic, and medial orbitofrontal cortex) evoked a loss-of-control phenotype, i.e., a substantial increase in the number of shocks incurred, a decrease in success trials, without major changes in omissions or latencies (Fig. 1b, left). This indicates that control over behavior under threat of punishment is governed by a neural network with the medial PFC as a core component, with a possible gradient across the dorsoventral axis. The effects of medial PFC inactivation on behavioral control are in contrast to earlier findings, that have shown that inactivation of the medial PFC does not affect impulsive choice, has mixed effects on impulsive action in the 5CSRTT, and has no effects on response inhibition in the stop-signal task (Cardinal, 2006; Winstanley et al., 2006; Pattij and Vanderschuren, 2008). The fact that our model involves the weighing of threat signals and the expectation of explicit punishment against the presence and consumption of palatable food, likely implicates the medial PFC (Sotres-Bayon and Quirk, 2010; Burgos-Robles et al., 2013; Jean-Richard-Dit-Bressel et al., 2018) to a larger extent than conventional impulse control tasks.

In contrast to the medial PFC, we observed a phenotype after pharmacological inactivation of the lateral orbitofrontal cortex that is reminiscent of a reduction in task engagement. As such, we observed an increase in omissions, which came, during stimulus trials, at the expense of the number of success trials, with an additional increase in pellet retrieval latencies. This observation was rather unexpected, given that the lateral orbitofrontal cortex has not clearly been implicated in incentive motivation or attention (Cardinal et al., 2002; Stalnaker et al., 2015; Izquierdo, 2017). One explanation for a reduction in task engagement could be that the animals were less able to comprehend task structure after inactivation, consistent with recent theories of a role for the lateral orbitofrontal cortex in guiding task execution by keeping a cognitive map of task structure (Wilson et al., 2014; Stalnaker et al., 2015). We speculate that inactivation may, as a result, impair the animal's ability to predict the timing of reward delivery or to link the foot shock to reward approach, thereby inducing task disengagement.

Inactivation of the basolateral amygdala evoked a phenotype that matched our hypothesized phenotype of a failure to retrieve stimulus value (Fig. 1 $b$, middle). Thus, after infusion of baclofen and muscimol, we observed a dramatic increase in the number of shock trials, without effects on omissions or behavior during no- stimulus trials. Given that the latency of pellet retrieval in success trials was also increased, we speculate that animals did not comprehend the offset of the threat signal, suggesting that animals were not able to retrieve the value of the audiovisual stimulus. As such, the animals behaved as if no threat signal was presented during stimulus trials, and entered the food port during the stimulus, thus receiving foot shock punishment, on the vast majority of trials. These data are consistent with a wealth of literature that shows an involvement of the basolateral amygdala in responding to a conditioned cue (Davis, 1997; Cardinal et al., 2002; Barad et al., 2006; Janak and Tye, 2015), thereby evoking behavior that is ostensibly fearless and punishment insensitive.

After pharmacological inactivation of the ventral striatum, the animals behaved as if they were less engaged in the task. We observed an increased number of omissions, and a reduced number of rewards collected, even during no-stimulus trials. Inactivation of the dorsomedial, and perhaps also dorsolateral, part of the striatum showed an attenuated version of this phenotype. These findings are consistent with the view that the motivational processes that are important for task performance primarily depend on ventral striatal circuits (Voorn et al., 2004; Floresco, 2015). It must be noted that we pharmacologically inactivated a relatively anterior part of the dorsal striatum, and there is evidence of a functional-anatomical gradient across its anteroposterior axis (Reynolds and Berridge, 2001; Pan et al., 2010; Mestres-Missé et al., 2012). For example, goal-directed behavior is shown to be dependent on the posterior, but not anterior, region of the dorsomedial striatum (Yin et al., 2005). It might therefore be the case that behavioral control is mediated by the dorsal striatum, but that this process takes place in its posterior parts, especially given that the absence of effects on the absolute number of shock trials challenges the classic view of the basal ganglia as part of the final common pathway of motoric $\mathrm{Go} /$ NoGo responses (Aron et al., 2007; Humphries and Prescott, 2010).

In sum, using a novel behavioral control task in rats, we show that behavioral inhibition is dependent on a network of corticolimbic areas, with the ventromedial PFC at its core, aided by striatal and orbitofrontal regions involved in task engagement, and the basolateral amygdala to encode the value of relevant conditioned stimuli. Our data provide an important step in the dissection of the brain circuits involved in behavioral inhibition, and hence contribute to the understanding of behaviors that are associated with poor control over behavior, including binge eating and drug abuse.

\section{References}

Ambroggi F, Ghazizadeh A, Nicola SM, Fields HL (2011) Roles of nucleus accumbens core and shell in incentive-cue responding and behavioral inhibition. J Neurosci 31:6820-6830.

Aron AR, Durston S, Eagle DM, Logan GD, Stinear CM, Stuphorn V (2007) Converging evidence for a fronto-basal-ganglia network for inhibitory control of action and cognition. J Neurosci 27:11860-11864.

Barad M, Gean PW, Lutz B (2006) The role of the amygdala in the extinction of conditioned fear. Biol Psychiatry 60:322-328.

Bari A, Robbins TW (2013) Inhibition and impulsivity: behavioral and neural basis of response control. Prog Neurobiol 108:44-79.

Burgos-Robles A, Bravo-Rivera H, Quirk GJ (2013) Prelimbic and infralimbic neurons signal distinct aspects of appetitive instrumental behavior. PLoS One 8:e57575.

Cardinal RN (2006) Neural systems implicated in delayed and probabilistic reinforcement. Neural Netw 19:1277-1301.

Cardinal RN, Parkinson JA, Hall J, Everitt BJ (2002) Emotion and motivation: the role of the amygdala, ventral striatum, and prefrontal cortex. Neurosci Biobehav Rev 26:321-352. 
Carli M, Robbins TW, Evenden JL, Everitt BJ (1983) Effects of lesions to ascending noradrenergic neurones on performance of a 5-choice serial reaction task in rats; implications for theories of dorsal noradrenergic bundle function based on selective attention and arousal. Behav Brain Res 9:361-380.

Cooper A, Barnea-Ygael N, Levy D, Shaham Y, Zangen A (2007) A conflict rat model of cue-induced relapse to cocaine seeking. Psychopharmacology 194:117-125.

Dalley JW, Robbins TW (2017) Fractionating impulsivity: neuropsychiatric implications. Nat Rev Neurosci 18:158-171.

Dalley JW, Everitt BJ, Robbins TW (2011) Impulsivity, compulsivity, and top-down cognitive control. Neuron 69:680-694.

Davis M (1997) Neurobiology of fear responses: the role of the amygdala. J Neuropsychiatry Clin Neurosci 9:382-402.

Eagle DM, Robbins TW (2003) Lesions of the medial prefrontal cortex or nucleus accumbens core do not impair inhibitory control in rats performing a stop-signal reaction time task. Behav Brain Res 146:131-144.

Evenden JL, Ryan CN (1996) The pharmacology of impulsive behaviour in rats: the effects of drugs on response choice with varying delays of reinforcement. Psychopharmacology 128:161-170.

Floresco SB (2015) The nucleus accumbens: an interface between cognition, emotion, and action. Annu Rev Psychol 66:25-52.

Humphries MD, Prescott TJ (2010) The ventral basal ganglia, a selection mechanism at the crossroads of space, strategy, and reward. Prog Neurobiol 90:385-417.

Izquierdo A (2017) Functional heterogeneity within rat orbitofrontal cortex in reward learning and decision making. J Neurosci 37:10529-10540.

Janak PH, Tye KM (2015) From circuits to behaviour in the amygdala. Nature 517:284-292.

Jean-Richard-Dit-Bressel P, Killcross S, McNally GP (2018) Behavioral and neurobiological mechanisms of punishment: implications for psychiatric disorders. Neuropsychopharmacology 43:1639-1650.

Jenkins TN, Warner LH, Warden CJ (1926) Standard apparatus for the study of animal motivation. J Comp Psychol 6:361-382.

Marchant NJ, Khuc TN, Pickens CL, Bonci A, Shaham Y (2013) Contextinduced relapse to alcohol seeking after punishment in a rat model. Biol Psychiatry 73:256-262.

McFarland K, Kalivas PW (2001) The circuitry mediating cocaine-induced reinstatement of drug-seeking behavior. J Neurosci 21:8655-8663.

Mestres-Missé A, Turner R, Friederici AD (2012) An anterior-posterior gradient of cognitive control within the dorsomedial striatum. Neuroimage 62:41-47.

Nederkoorn C, Braet C, Van Eijs Y, Tanghe A, Jansen A (2006) Why obese children cannot resist food: the role of impulsivity. Eat Behav 7:315-322.

Nederkoorn C, Houben K, Hofmann W, Roefs A, Jansen A (2010) Control yourself or just eat what you like? Weight gain over a year is predicted by an interactive effect of response inhibition and implicit preference for snack foods. Health Psychol 29:389-393.

Orsini CA, Trotta RT, Bizon JL, Setlow B (2015) Dissociable roles for the basolateral amygdala and orbitofrontal cortex in decision-making under risk of punishment. J Neurosci 35:1368-1379.

Pan WX, Mao T, Dudman JT (2010) Inputs to the dorsal striatum of the mouse reflect the parallel circuit architecture of the forebrain. Front Neuroanat $4: 147$.

Pattij T, Vanderschuren LJ (2008) The neuropharmacology of impulsive behaviour. Trends Pharmacol Sci 29:192-199.

Reynolds SM, Berridge KC (2001) Fear and feeding in the nucleus accumbens shell: rostrocaudal segregation of GABA-elicited defensive behavior versus eating behavior. J Neurosci 21:3261-3270.

Robbins TW (2002) The 5-choice serial reaction time task: behavioural pharmacology and functional neurochemistry. Psychopharmacology 163:362-380.

Simon NW, Gilbert RJ, Mayse JD, Bizon JL, Setlow B (2009) Balancing risk and reward: a rat model of risky decision making. Neuropsychopharmacology 34:2208-2217.

Sotres-Bayon F, Quirk GJ (2010) Prefrontal control of fear: more than just extinction. Curr Opin Neurobiol 20:231-235.

Stalnaker TA, Cooch NK, Schoenbaum G (2015) What the orbitofrontal cortex does not do. Nat Neurosci 18:620-627.

van der Plasse G, van Zessen R, Luijendijk MC, Erkan H, Stuber GD, Ramakers GM, Adan RA (2015) Modulation of cue-induced firing of ventral tegmental area dopamine neurons by leptin and ghrelin. Int J Obes 39:1742-1749.

Verharen JPH, de Jong JW, Roelofs TJM, Huffels CFM, van Zessen R, Luijendijk MCM, Hamelink R, Willuhn I, den Ouden HEM, van der Plasse G, Adan RAH, Vanderschuren LJMJ (2018) A neuronal mechanism underlying decision-making deficits during hyperdopaminergic states. Nat Commun 9:731.

Voorn P, Vanderschuren LJ, Groenewegen HJ, Robbins TW, Pennartz CM (2004) Putting a spin on the dorsal-ventral divide of the striatum. Trends Neurosci 27:468-474.

Wilson RC, Takahashi YK, Schoenbaum G, Niv Y (2014) Orbitofrontal cortex as a cognitive map of task space. Neuron 81:267-279.

Winstanley CA, Eagle DM, Robbins TW (2006) Behavioral models of impulsivity in relation to ADHD: translation between clinical and preclinical studies. Clin Psychol Rev 26:379-395.

Yin HH, Ostlund SB, Knowlton BJ, Balleine BW (2005) The role of the dorsomedial striatum in instrumental conditioning. Eur J Neurosci 22: 513-523. 